Article

\title{
There Are No Conformal Einstein Rescalings of Pseudo-Riemannian Einstein Spaces with $n$ Complete Light-Like Geodesics
}

\author{
Josef Mikeš ${ }^{1}\left(\mathbb{D}\right.$, Irena Hinterleitner ${ }^{2}(\mathbb{D})$ and Nadezda Guseva ${ }^{3, *}$ \\ 1 Department of Algebra and Geometry, Palacky University, 17. listopadu 12, 77146 Olomouc, Czech Republic \\ 2 Department of Mathematics, Faculty of Civil Engineering, Brno University of Technology, \\ 60190 Brno, Czech Republic \\ 3 Department of Geometry, Moscow Pedagogical State University, 1/1 M. Pirogovskaya Str., \\ 119991 Moscow, Russian \\ * Correspondence: josef.mikes@upol.cz; Tel.: +420-731-579-395
}

Received: 31 July 2019; Accepted: 29 August 2019; Published: 1 September 2019

\begin{abstract}
In the present paper, we study conformal mappings between a connected $n$-dimension pseudo-Riemannian Einstein manifolds. Let $g$ be a pseudo-Riemannian Einstein metric of indefinite signature on a connected $n$-dimensional manifold $M$. Further assume that there is a point at which not all sectional curvatures are equal and through which in linearly independent directions pass $n$ complete null (light-like) geodesics. If, for the function $\psi$ the metric $\psi^{-2} g$ is also Einstein, then $\psi$ is a constant, and conformal mapping is homothetic. Note that Kiosak and Matveev previously assumed that all light-lines were complete. If the Einstein manifold is closed, the completeness assumption can be omitted (the latter result is due to Mikeš and Kühnel).
\end{abstract}

Keywords: pseudo-Riemannian manifold; Einstein manifold; concircular vector field; conformal mapping; light-like geodesic; complete geodesic

\section{Introduction}

As is well known, Einstein spaces play a very important role in the general theory of relativity. The conformal mappings of these spaces has been studied since 1920 by Brinkmann [1], see [2,3]. Brinkmann proved that this task is closely related to the existence of concircular vector fields.

In 1944, Yano [4-7] introduced term a concircular vector field $\xi$, which satisfies $\nabla \xi=\varrho \cdot I d$, where $\nabla$ is affine connection. The existence of concircular vector fields "as a whole" was studied in papers [4-13].

A lot of work has been devoted to special mappings of Einstein spaces, such as [2,3,8,9,14-24].

Kühnel and Rademacher in [21] presented some results on Einstein spaces with a conformal group, and also conformal mappings. Many of these results are formulated for (geodesical) complete Einstein spaces.

In our paper, we find a generalization of results by Kiosak and Matveev [19], see Remark 4.

\section{Main Results}

We suppose that domain $V$ of $n$-dimensional manifold $M$ is connected and one of the following condition holds: (1) $V$ is without a boundary; (2) $\partial V$ is the Lipschitz boundary, i.e., domain $V$ lies on one side of $\partial V$, see [25], p. 46; (3) $V$ is the weakly Lipschitz domain, see [26]. 
The following theorem is proved in our paper.

Theorem 1. Let $g$ be a pseudo-Riemannian Einstein metric of indefinite signature on a domain $V$ of $n$-dimensional manifold $M$. Further assume that there is a point at which not all sectional curvatures are equal and through which in linearly independent directions pass $n$ complete null (light-like) geodesics. If, for the function $\psi$, the metric $\psi^{-2} g$ is also Einstein, then $\psi$ is a constant.

Remark 1. Complete geodesics condition in Theorem 1 can be substituted for closed. (This term is obviously used in Riemannian geometry).

Remark 2. Evidently, the dimension $n$ in Theorem 1 is more than 3. For $n=2$, it is trivial, and for $n=3$ any Einstein space has the constant curvature.

Remark 3. In the four-dimensional Lorentz case, conformal Einstein rescalings of Einstein metrics were described by Brinkmann [1].

Remark 4. Kiosak and Matveev [19] proved the following theorem (see comments in [21]).

Theorem 2. Let $g$ be a light-line-complete pseudo-Riemannian Einstein metric of indefinite signature (i.e., for no constant $c$ the metric $c \cdot g$ is Riemannian) on a connected $(n>2)$-dimensional manifold $M$. Assume that, for the nowhere vanishing function $\psi$, the metric $\psi^{-2} g$ is also Einstein. Then, $\psi$ is a constant.

Remark 5. Theorems 1 and 2 fail for Riemannian metrics (even if we replace light-line completeness by usual completeness)-Möbius transformations of the standard round sphere and the stereographic map of the punctured sphere to the Euclidean space are conformal nonhomothetic mappings. One can construct other examples on warped Riemannian manifolds, see ([20] Theorem 21).

Remark 6. By Theorem 1, pseudo-Riemannian Einstein metrics of indefinite signature with $n$ complete light-line do not admit nonhomothetic conformal complete vector fields. The Riemannian version of this result is due to Yano and Nagano [13]. Moreover, the assumption that the metric is Einstein can be omitted (by the price of considering only essential conformal vector fields): as it was proved by Alekseevskii [27], Ferrand [28] and Schoen [29], a Riemannian manifold admitting an essential complete vector field is conformally equivalent to the round sphere or the Euclidean space. It is still not known whether the last statement (sometimes called Lichnerowicz-Obata conjecture) can be extended to the pseudo-Riemannian case, see [30] for a counterexample in the $C^{1}$-smooth category, and [31,32] for a good survey on this topic.

Remark 7. A partial case of Theorem 1 is ([21] Theorem 2.2), in which it is assumed that both metrics are complete. This extra assumption is very natural in the context of [21] since the paper is dedicated to the classification of conformal vector fields; moreover, Theorem 2.2 is not the main result of the paper. It is not clear whether, in the proof of ([21] Theorem 2.2), the assumption that the second metric is complete could be omitted.

\section{Proof of Theorem 1}

It is well known (see for example ([1] Equation (2.21)), ([20] Lemma 1) or [2,3,15,23]) that the Ricci curvatures $R_{i j}$ and $\bar{R}_{i j}$ of two conformally equivalent metrics $g$ and $\bar{g}=\psi^{-2} g=e^{-2 \varphi} g$ on the domain $V$ of manifold $M$ are related by

$$
\bar{R}_{i j}=R_{i j}+\left(\Delta \varphi-(n-2)\|\nabla \varphi\|^{2}\right) g_{i j}+\frac{n-2}{\psi} \nabla_{i} \nabla_{j} \psi
$$


We rewrite Equation (1) to the following form:

$$
\nabla_{i} \nabla_{j} \psi=\varrho g_{i j}
$$

where $\varrho$ is a function on the domain $V$. It is evident that $\xi^{h}=\nabla_{\alpha} \psi g^{h \alpha}$ ( $g^{i j}$ are components of the inverse matrix $g_{i j}$ ) is a concircular vector field.

Kazdan and deTurck [33], see [14], proved that locally there exists an analytic coordinate system $x$ in an Einstein manifold, i.e., the components $g_{i j}(x)$ are real analytic functions. Therefore, the functions $\psi(x)$ and $\varrho(x)$ that satisfy Equation (2) are also real analytic, see [18], ([23] p. 143).

Consider a null (light-like) geodesic $\gamma(t)$ of the metric $g$. Since the geodesic $\gamma(t)$ is complete, $\gamma(t)$ satisfies equation $\nabla_{\dot{\gamma}} \dot{\gamma}=0$ on the whole $\mathbb{R}$, where $\dot{\gamma}$ is the velocity vector of $\gamma$. "Light-like" means that $g(\dot{\gamma}(t), \dot{\gamma}(t))=g_{i j} \dot{\gamma}^{i}(t) \dot{\gamma}^{j}(t)=0$. It is well known that, if this property is fulfilled in one point, then it is fulfilled at every point of the geodesic.

We calculate $\frac{d^{2}}{d t^{2}} \psi(\gamma(t))=\nabla_{i} \nabla_{j} \psi(\gamma(t)) \dot{\gamma}^{i} \dot{\gamma}^{j}$. Since $R_{i j}, \bar{R}_{i j}$ and $\bar{g}_{i j}$ are proportional to $g_{i j}$, therefore, from Equation (1), we obtain $\frac{d^{2}}{d t^{2}} \psi(\gamma(t))=0$. Evidently, $\psi(\gamma(t))=$ const $_{1} \cdot t+$ const. Since by assumptions the function $\psi$ is defined on the whole $\mathbb{R}$ and is equal to zero at no point, we have $\psi=$ const along complete light-like geodesics. See, for example, [19].

It is known, for example ([23] p. 115), that, from the Ricci identity

$$
\left(\nabla_{k} \nabla_{j}-\nabla_{j} \nabla_{k}\right) \psi_{i}=\psi_{h} R_{i j k}^{h}
$$

where $R_{i j k}^{h}$ are components of the Riemann tensor curvature and $\psi_{i}=\nabla_{i} \psi$; from Equation (2), we obtain

$$
\psi_{h} R_{i j k}^{h}=g_{i j} \nabla_{k} \varrho-g_{i k} \nabla_{j} \varrho
$$

After contracting (3) with $g^{i j}$, we get

$$
\nabla_{i} \varrho=-\frac{R}{n(n-1)} \psi_{i}
$$

where $R=R_{i j} g^{i j}$ is the scalar curvature on $V$; evidently, $R$ is a constant.

We found a linear Cauchy system of differential equations in covariant derivatives

$$
\begin{aligned}
\nabla_{i} \psi & =\psi_{i}, \\
\nabla_{i} \psi_{j} & =\varrho g_{i j}, \\
\nabla_{i} \varrho & =-\frac{R}{n(n-1)} \psi_{i},
\end{aligned}
$$

with respective unknown functions $\psi(x), \psi_{i}(x)$ and $\varrho(x)$.

This system has at most one solution on $V$ which meets the requirements for the boundary $\partial V$ for the Cauchy initial conditions (in a detail see [23], pp. 130-133)

$$
\psi\left(x_{0}\right)=\psi^{0}, \quad \psi_{i}\left(x_{0}\right)=\psi_{i}^{0}, \quad \varrho\left(x_{0}\right)=\varrho^{0} .
$$

Evidently, for initial conditions $\psi\left(x_{0}\right)=\psi^{0}, \psi_{i}\left(x_{0}\right)=0, \varrho\left(x_{0}\right)=0$, Equation (4) has a unique trivial solution $\psi(x)=\psi^{0}, \psi_{i}(x)=0, \varrho(x)=0$ for all $x \in V$. 
The condition (3) with (4) has the following form:

$$
\psi_{h} Y_{i j k}^{h}=0,
$$

where $\delta_{i}^{h}$ is the Kronecker symbol and $Y_{i j k}^{h}$ is the Yano tensor

$$
Y_{i j k}^{h}=R_{i j k}^{h}-\frac{R}{n(n-1)}\left(g_{i j} \delta_{k}^{h}-g_{i k} \delta_{j}^{h}\right) .
$$

Deriving (5) and applying Equation (4), we get

$$
\varrho Y_{l i j k}+\psi_{h} \nabla_{l} Y_{i j k}^{h}=0,
$$

where $Y_{l i j k}=g_{l h} Y_{i j k}^{h}$.

Let $x_{0} \in V$ be the point from Theorem 1, which, from the non-identical section curvature in this point, follows $Y_{h i j k}\left(x_{0}\right) \not \equiv 0$. Due to $n$ complete light-like geodesics go through at $x_{0}$, the function $\psi(x)$ is constant along those geodesics. In these null (isotropic) directions $d \psi(\gamma(t)) / d t=0$, and because these $n$ directions form a basis, we obtain $\psi_{i}\left(x_{0}\right)=0$. From (7), it follows $\varrho\left(x_{0}\right)=0$, and system (4) has only trivial solutions $\psi(x)=\psi\left(x_{0}\right)=$ const. Because $V$ is connected (and meets the requirements for the boundary $\partial V)$, this local solution may be extended on all $V$.

\section{Example of Non-Trivial Mappings with $n$-Complete Light-Like Geodesics Go through at the Point}

Let $M$ be a part of an $n$-dimensional pseudo-Euclidean space with Cartesian coordinates $\left(x^{1}, x^{2}, \ldots, x^{n}\right)$ and metric $g=\sum_{i=1}^{n} e_{i}\left(x^{i}\right)^{2}, e_{i}= \pm 1$, which is defined by inequality

$$
\frac{1}{2} c \sum_{i=1}^{n} e_{i}\left(x^{i}\right)^{2}+\psi^{0}>0
$$

where $c$ and $\psi^{0}$ are positive constants.

Any light-like geodesics with go through at point $(0,0, \ldots, 0)$ is complete on $M$.

The function

$$
\psi=\frac{1}{2} c \sum_{i=1}^{n} e_{i}\left(x^{i}\right)^{2}+\psi^{0}
$$

is a positive solution of Equation (2) on $M$, and metric $\psi^{-2} g$ is regular.

Evidently, $g$ and $\psi^{-2} g$ are Einstein metrics.

Author Contributions: All authors contributed equally and significantly in writing this article.

Funding: This research received no external funding.

Acknowledgments: The paper was supported by the project IGA PrF 2019015 Palacky University Olomouc and No. LO1408 "AdMaS UP"-Advanced Materials, Structures and Technologies supported by Ministry of Education, Youth and Sports under the "National Sustainability Programme I" of the Brno University of Technology.

Conflicts of Interest: The authors declare no conflict of interest. 


\section{References}

1. Brinkmann, H.W. Einstein spaces which mapped conformally on each other. Math. Ann. 1925, 94, 119-145. [CrossRef]

2. Petrov, A.Z. New Methods in the General Theory of Relativity; Nauka: Moscow, Russia, 1966.

3. Petrov, A.Z. Einstein Spaces; Pergamon Press: Oxford, UK; New York, NY, USA, 1969.

4. Yano, K. Concircular geometry I: Concircular transformations. Proc. Imp. Acad. 1940, 16, 195-200.

5. Yano, K. Concircular geometry II: Integrability conditions of $\rho_{\mu v}=\phi g_{\mu v}$. Proc. Imp. Acad. 1940, $16,442-448$.

6. Yano, K. Concircular geometry III: Theory of curves. Proc. Imp. Acad. 1940, 9, 442-448.

7. Yano, K. Concircular geometry IV: Theory of subspaces. Proc. Imp. Acad. 1940, 10, 505-511.

8. Mikeš, J. Geodesic mappings of affine-connected and Riemannian spaces. J. Math. Sci. (N. Y.) 1996, 78, 311-333.

9. Mikeš, J. On concircular vector fields "in large" on compact Riemannian spaces. Odessk. Univ. Kiev Arch. 1988, 615-Uk88. (In Russian)

10. Mikeš, J.; Chodorová, M. On concircular and torse-forming vector fields on compact manifolds. Acta Math. Acad. Paedagog. Nyházi. (N.S.) 2010, 26, 329-335.

11. Mikeš, J.; Radulović, Ž. On concircular and torse-forming vector fields "in the large". Math. Montisnigri 1995, 4, 43-54.

12. Yano, K.; Bochner, S. Curvature and Betti Numbers; Princeton University Press: Princeton, NJ, USA, 1953.

13. Yano, K.; Nagano, T. Einstein spaces admitting a one-parameter group of conformal transformations. Ann. Math. 1959, 69, 451-461. [CrossRef]

14. Besse, A.L. Einstein Manifolds; Springer: Berlin/Heidelberg, Germany, 1987.

15. Eisenhart, L.P. Riemannian Geometry; Princeton Univ. Press: Princeton, NJ, USA, 1949.

16. Fialkow, A. Conformals geodesics. Trans. Am. Math. Soc. 1939, 45, 443-473. [CrossRef]

17. Formella, S.; Mikeš, J. Geodesic mappings of Einstein spaces. Szczec. Rocz. Nauk. Ann. Sci. Stetin. 1994, 9, 31-40.

18. Hinterleitner, I.; Mikeš, J. Geodesic mappings and Einstein spaces. Geometric methods in physics. Birkhäuser/Springer. Trends Math. 2013, 19, 331-335.

19. Kiosak, V.; Matveev, V.S. There are no conformal Einstein rescalings of complete pseudo-Riemannian Einstein metrics. C. R. Math. Acad. Sci. Paris 2009, 347, 1067-1069. [CrossRef]

20. Kühnel, W. Conformal transformations between Einstein spaces. In Conformal Geometry (Bonn, 1985/1986); Aspects of Mathematics; Vieweg: Braunschweig, Germany, 1988; Volume 12, pp. 105-146.

21. Kühnel, W.; Rademacher, H.-B. Einstein spaces with a conformal group. Results Math. 2009, 56, 421-444. [CrossRef]

22. Mikeš, J. Geodesic mappings of Einstein spaces. Math. Notes 1980, 28, 428-431.

23. Mikeš, J.; Bácsó, S.; Berezovski, V.; Chepurna, E.; Chodorová, M.; Chudá, H.; Formella, S.; Gavrilchenko, M.; Haddad, M.; Hinterleitner, I.; et al. Differential Geometry of Special Mappings; Palacky University Press: Olomouc, Czech Republic, 2019.

24. Mikeš, J.; Kiosak, V.A. On geodesic mappings of four dimensional Einstein spaces. Odessk. Univ. Moscow Arch. VINITI 9.4.82 1982, 1678-1682._28. [CrossRef]

25. Fučík, S.; Kufner, A. Nonlinear Differential Equations; TKI, SNTL: Praha, Slovakia, 1978.

26. Dacorogna, B. Introduction to the Calculus of Variations; Imperial College Press: London, UK, 2004.

27. Alekseevskii, D.V. Groups of conformal transformations of Riemannian spaces. Mat. Sbornik 1972, 89, $285-301$. [CrossRef]

28. Ferrand, J. The action of conformal transformations on a Riemannian manifold. Math. Ann. 1996, 304, $277-291$. [CrossRef]

29. Schoen, R. On the conformal and CR automorphism groups. Geom. Funct. Anal. 1995, 5, 464-481. [CrossRef]

30. Leitner, F. Twistor spinors with zero on Lorentzian 5-space. Comm. Math. Phys. 2007, 275, 587-605. [CrossRef]

31. Frances, C. Sur le groupe d'automorphismes des géométries paraboliques de rang 1. Ann. Sci. Ecole Norm. Supérieure 2007, 40, 741-764. Available online: http://www.math.u-psud.fr/?frances/cartan-english6.pdf (accessed on 1 August 2019 ). [CrossRef] 
32. Frances, C. Essential conformal structures in Riemannian and Lorentzian geometry. In Recent Develop-Ments in Pseudo-Riemannian Geometry; ESI Lectures in Mathematics and Physics; European Mathematical Society: Zürich, Switzerland, 2008; Volume 4, pp. 231-260.

33. DeTurck, D.M.; Kazdan, J.L. Some regularity theorems in Riemannian geometry. Ann. Sci. Éc. Norm. Supér. 1981, 14, 249-260. [CrossRef] article distributed under the terms and conditions of the Creative Commons Attribution (CC BY) license (http:/ / creativecommons.org/licenses/by/4.0/). 